\title{
The Gulf War and illness by association
}

\author{
Eamonn Ferguson* and Helen J. Cassaday \\ School of Psychology, University of Nottingham, UK
}

\begin{abstract}
The symptoms of Gulf War Syndrome (GWS) closely correspond to the physiological and behavioural sequelae of an interleukin-1 (IL-1) mediated sickness response. We propose that this response can account for the variability in its symptomatology. Furthermore, the persistence of GWS can be accounted for by an associative process, whereby the smells/tastes of war become linked with the physiological reaction to environmental stressors encountered in the Gulf and/or the 'cocktail' of drugs given to the soldiers. As required, the IL-1 response is conditionable.
\end{abstract}

On 2 August 1990 Iraq invaded Kuwait. Between August 1990 and March 1991, supported by UN resolution 660, land and air troops were deployed in the Persian Gulf. Operation Desert Storm commenced on 16 January 1991 with 39 days of air strikes followed by 4 days of a ground war. On returning from the Persian Gulf numerous veterans began to show signs of illness that they attributed to their experience in the Gulf. The symptoms were diverse including nausea, headaches, malaise, depression, rashes, memory, sexual and sleep problems (Department of Defence, 1996; see also Tables 1 and 3). This has led some to question the plausibility of a unitary Gulf War Syndrome (GWS) and talk instead of a set of Gulf War Syndromes (Milner, Axelrod, Pasquantonio \& Sillanpaa, 1994; Table 1). However, as well as being diverse, the associated symptoms are also characterized by their persistence. Therefore, any complete account of GWS (or syndromes) must attempt to account for this diversity and persistence.

Accounting for both the diversity and the persistence of symptoms seen in GWS poses a unique challenge to researchers attempting to develop any model of perceptions of illness. Development of a theoretical understanding of syndromes as complex as GWS may also help a move towards more general models of symptom persistence and variability in other disease states. As a first step in developing this understanding, we review the evidence for the construct of Gulf War Syndrome(s). We then explore the evidence for one of the main theories of GWS, chronic organophosphate-induced delayed polyneuropathy (OPIDP) (Haley \& Kurt, 1997). However, the OPIDP theory has a number of shortcomings as a complete account of the data. Finally we present a psycho-neuroendocrine model that can account for the variability in symptoms (following Maier \& Watkins, 1998) and show how associative processes could account for the persistence of symptoms on return from the Gulf.

* Requests for reprints should be addressed to Eamonn Ferguson, School of Psychology, University of Nottingham, University Park, Nottingham NG7 2RD, UK (e-mail: ef@psyc.nott.ac.uk). 


\section{Symptom diversity: Single or multiple syndromes?}

Recently evidence has been presented questioning the very existence of GWS (Ismail et al., 1999). However, the interpretation of that evidence has itself been questioned (Ferguson, 1999), and the veterans are most certainly ill (Unwin et al., 1999).

Of a sample of 249 veterans studied by Haley, Kurt \& Hom (1997b), 115 (46\%) believed that their illness was definitely caused by being in the Gulf (they claimed $100 \%$ certainty), $45(18 \%)$ that it was more than $50 \%$ probable and $14(6 \%)$ that it was possible $(<50 \%$ probable). Only one of this sample thought that the experience of illness was not connected with being in the Gulf.

GWS is marked by a wide variety of symptomatology (Department of Defence, 1996; Tables 1 and 3). This has lead some to suggest that in fact it is not a 'real' medical syndrome as such, but may consist of a variety of syndromes (Milner et al., 1994). Indeed, factor analytic work has suggested that, amongst veterans presenting with GWS, there may be six symptom clusters/syndromes or factors (Haley et al., 1997b). These Gulf War Syndromes and their defining symptom features are described in Table 1.

Table 1. The six Gulf War Syndromes identified by Haley et al. (1997b)

Syndrome

Symptoms

1. Cognitive impairment

2. Confusion-ataxia

3. Arthro-myo-neuropathy

4. Phobia-apraxia

5. Fever adenopathy

6. Weakness-incontinence
Distractibility, difficulty remembering, depression, middle and terminal insomina, fatigue (daytime sleeping), confused thoughts and migraine.

Problems with reading, writing and spelling, getting confused, disorientation, problems with balance, liver disease, sexual impotence.

Generalized joint and muscle pain, difficulty lifting heavy objects, fatigue (muscle exhaustion), tingling and numbness in the extremities.

Nausea, numbness and tingling (trunk not extremities), faintness, chest pains (when in tight situations with no immediate escape).

Swollen lymph glands (various sites), fever sometimes with night sweats.

Problems with bowel and bladder movements, tingling in face, tongue and lips.

While Haley et al. (1997b) identified six 'syndromes', in subsequent work they have gone on to explore only the first three and ignore the last three, arguing that the first three syndromes are the primary syndromes. In any case, the application of exploratory factor analysis of self-reported symptoms in itself is not sufficient to identify these as a set of syndromes (see Kendell, 1989). For example, responses on such measures may be affected by other psychological dispositions. Indeed, Haley et 
al. (1997b) indicate that, compared to controls, their sample of veterans scored higher on scales designed to assess somatization and general health concerns. Further, extraction heuristics used in factor analysis to decide on the optimal number of factors to extract vary with respect to their accuracy (Ferguson \& Cox, 1993; Zwick \& Velicer, 1986). Haley et al. (1997b) used the Scree test to identify the optimal number of factors, which, whilst reasonably accurate, is not necessarily the most reliable (Zwick \& Velicer, 1986). Further, Haley et al.'s (1997b) unrotated Scree plot is dominated by one large eigenvalue (approximately 11.00), the next largest eigenvalue being around 2.0. This pattern in itself may suggest a single general factor (cf. Gorsuch, 1983). One extraction heuristic, parallel analysis (PA) has been shown through Monte Carlo studies to approach $100 \%$ accuracy with high factor saturation (average loading .8) (Zwick \& Velicer, 1986). PA compares the observed eigenvalues to the average eigenvalues produced by the factor analysis of a series of random data sets, based on the same sample size and number of items as the observed data set (Horn, 1965). The number of factors is indicated when the observed eigenvalues are larger than the eigenvalues produced by the random data sets. Haley et al. (1997b) provided the Scree plot for their analysis from which it was possible to obtain an accurate estimate of their observed eigenvalues by reading the $\mathrm{Y}$ axis of their $\mathrm{Scree}$ plot. Thus it was possible to generate a series of random data sets (10 in all) based on the same sample size as Haley et al. (1997b) and the same number of variates. Table 2 provides a direct comparison of the observed and average eigenvalues from the random data simulation.

Table 2. Parallel analysis of the Haley et al. (1997b) data

Observed eigenvalues

(Haley et al., 1997b) Random eigenvalue (SD)

\begin{tabular}{ll}
\hline 11 & $2.03(0.09)$ \\
2.1 & $1.91(0.04)$ \\
1.75 & $1.84(0.02)$ \\
1.45 & $1.79(0.03)$ \\
1.4 & $1.72(0.03)$ \\
1.38 & $1.66(0.02)$ \\
1.00 & $1.61(0.03)$ \\
0.9 & $1.56(0.02)$ \\
0.85 & $1.51(0.03)$ \\
0.8 & $1.46(0.04)$ \\
\hline
\end{tabular}

As can be seen from Table 2 the observed eigenvalues exceed the random eigenvalues only for the first two factors, and only just for the second factor. This analysis would indicate that six symptom clusters are probably an overestimate and a more parsimonious explanation based on one or perhaps two symptom clusters is equally (if not more) plausible. More credence could be given to six separable factors 
if the authors had reported the degree of correlation between the factors, and shown them to be essentially orthogonal. As it is, there is no evidence pertaining to this and it may well be the case that veterans scoring high on one factor may also score high on another. Further, within these six 'syndromes' Hom, Haley \& Kurt (1997) were unable to show any real variation in psychological profile across the groups.

Perhaps more importantly, to identify separable 'syndromes' conclusively, it is necessary to show that individuals with different diagnostic profiles vary systematically on a variety of objective medical tests. This is exactly what Haley et al. (1997 a) set out to do. To present this conclusively, however, it is necessary to show that cases are significantly different from controls (systematic differences between case and non-case groups) and that, within the generic group showing symptoms, one syndrome is distinguishable from another (systematic differences within the general category of case, cf. Kendell, 1989). As required, these authors were able to demonstrate between groups differences. Haley et al. (1997a) took 165 measures from their sample, but were only able to identify 35 tests on which cases and controls were different. Applying these 35 tests, cases 'appeared more impaired on 27 and controls on 8' (Haley et al., 1997a, p. 228). However, the results are less than conclusive with regards to the differences shown within the general category of case. Here the analysis showed little or no discrimination between veterans identified as having one 'syndrome' as opposed to another.

A final methodological problem with the Haley et al. (1997a) study arises from their sample sizes and the level at which alpha was set for statistical tests. For this study, the borderline $p$-value was set at .1. While this will mean that clinically significant differences that could otherwise be missed would not be overlooked (especially important given the low sample size of 5 to 13 participants), it does mean that 'differences' may also have been unduly exaggerated. If so, this weakens the evidence for separable 'syndromes'. The conclusion that, indeed, there may not be separable Gulf War Syndromes has previously been expressed without a full reexamination of the data (see Holden, 1997). In this section, therefore, we have tried to explore in more detail than previously reported, the problems with the idea of multiple syndromes.

Thus while the symptomatology of 'GWS' is wide-ranging, the evidence for various Gulf War Syndromes (from now on, also abbreviated to GWS for ease of exposition) is by no means conclusive. An account in terms of a single syndrome is more parsimonious. The challenge then becomes to identify a common causal linkage for that syndrome that is both (1) reasonably complete (providing some account of all the definitive symptoms) and (2) testable (giving further insight into the nature of GWS and/or suggesting useful treatment interventions). Ideally, an explanation specifying the biological mechanisms is required. One of the most widely cited accounts of GWS is the OPIDP model.

\section{The OPIDP model of GWS}

Irrespective of the status of illness following the Gulf War as a single syndrome or a variety of syndromes, one plausible account with supporting evidence is the notion 
that the symptoms result from a form of chronic organophosphate (OP) poisoning resulting in polyneuropathy (Haley \& Kurt, 1997; Hom et al., 1997; Jamal, Hansen, Apartopoulos \& Peden, 1996). OPIDP is believed to be associated with exposure to combinations of a variety of OPs and other cholinesterase-inhibiting chemicals. The clinical features of OPIDP are attributable to the inhibition, via phosphorylation, of an enzyme known as neuropathy target enzyme or neuropathy target esterase (NTE) (see Lotti, 1992; Lotti, Becker \& Aminoff, 1984, for reviews). This phosphorylation is a time-dependent process referred to as 'ageing' (Lotti, 1992). Further clinical features do not occur until $70 \%$ of NTE has been inhibited (see De Bleecker, De Reuck \& Willems, 1992; Jamal, 1995; Lotti, 1992). Clinically, there are some symptoms associated with acute cholinesterase inhibitor poisoning and later (chronic) OPIDP that resemble those reported in GWS (see De Bleecker et al., 1992). The acute form of OPIDP produces a set of symptoms that affect the cardiovascular, urinary, gastrointestinal and central nervous systems. These symptoms may be short-lived and are clinically managed by a variety of procedures (see De Bleecker et al., 1992). The chronic variant usually manifests between 1 and 3 weeks after exposure (De Bleecker et al., 1992; Jamal, 1995) and may produce permanent changes due to OPs binding to NTE throughout the nervous system. Chronic OPIDP of this nature has itself been referred to by Haley \& Kurt (1997) as a wide-ranging condition 'of central-peripheral, distal, sensory-motor axonopathy’ (see Haley \& Kurt, 1997, p. 231). These long-term OPIDP symptoms may result from two types of exposure: (1) acute (as described above) and (2) chronic long-term exposure to low doses of OPs (Jamal, 1995). In relation to the symptoms reported by Gulf War veterans, three conditions would have to be met to confirm the hypothesis that OPIDP is a complete or sufficient cause of GWS.

First, the model requires that those serving in the Persian Gulf were exposed to low level dosages of OPs for the period of the conflict, rather than presenting with acute OP poisoning. This distinction matters because the symptoms of acute OP poisoning are more severe than those generally seen in the chronic presentation. However, these obvious symptoms were not reported en masse by veterans during their time in the Persian Gulf (and acute OP poisoning can be clinically managed, see De Bleecker et al., 1992). While the possibility remains that some acute OP poisoning may have occurred and resulted in OPIDP, a low level chronic poisoning would provide a better explanation of how so many service personnel came to be affected. However, ' ... there has been no universal acceptance of the fact that organophosphate esters are capable of producing long-term chronic effects at doses below those causing acute clinical intoxication in humans' (Jamal, 1995, p. 90). Further, Jamal (1995) suggests that one study's failure to demonstrate significant differences between a group exposed to OPs and a control could have been due to the exposed group experiencing only a short duration exposure of 39 days. Whilst exposures of this duration may not be sufficient to produce OPIDP symptomatology, 39 days is about the same as the period of Operation Desert Storm, during which a significant proportion of the required OP exposure was likely to have occurred.

Secondly, does the spectrum of symptoms attributable to OPIDP correspond to 
the diversity of symptoms reported in veterans? The answer here is a partial 'yes'. De Bleecker et al. (1992) report symptoms relating to muscular weakness, pains in the legs and sensory disturbances. Jamal (1995) adds to this list gastrointestinal effects, headache, general weakness, insomnia and other neuropsychiatric symptoms. There is also some evidence that a variety of psychiatric and psychological symptoms may be attributable to acute (see Rosenstock, Keifer, Daniell, McConnell \& Claypoole, 1991) and chronic exposure to OPs (see Barnes, 1961; Gershon \& Shaw, 1961; Petty, 1958); however, the evidence is by no means conclusive (see Stroller, Krupinski, Christophers \& Blanks, 1965; Jamal, 1995, for a review).

Finally, it is necessary to demonstrate that those who served in the Gulf and report symptoms were more likely to have been exposed to OPs than those with otherwise comparable experiences who do not report symptoms. Haley \& Kurt (1997) addressed this question using a cross-sectional survey: participants self-reported the use of a variety of precautionary measures (e.g. flea collars, insect repellents etc.) that may contain acetylcholinesterase (AChE) inhibitors. These authors then investigated whether the reported use of these prophylactics placed veterans at greater risk of reporting symptoms clusters than those who did not report that they had used them. The results showed that retrospective reports of increased use of flea collars and insect repellents were more likely to be associated with high scores on each of the first three factors previously identified (cf. Haley et al., 1997 b). Although these authors tried to reduce recall bias as much as possible, it is still possible that these associations are biased by attributional processes and the desire to find meaning (cf. Skelton \& Pennebaker, 1982; Taylor, 1983). These biases are particularly likely to operate for those with 'health anxieties' (Elderkin-Thompson, Silver \& Waitzkin, 1998; Robins \& Kirmayer, 1991), and the data reported by Haley et al. (1997b) indicated that their cases had higher than average levels of health anxiety. Further, a case-control design would have provided stronger evidence as such a design would make it possible to show that those who did not report symptoms (the control group) also reported less use of AChE inhibitors, flea collars etc.

The balance of evidence suggests that the OPIDP model provides a general account of the variety of symptoms seen in GWS. It can also account for the fact that these adverse effects can be very long-lasting. In addition, there are further symptoms reported by veterans that are not a central part of the symptom complex described for OPIDP. For example, an account based on OPIDP does not readily explain liver problems and persistent pain sensitivity, increased levels of aggression, sexual problems and persistent fever. Problems with identifying both level and duration of exposure further limits the OPIDP model. The OPIDP account does provide a plausible account of a range of cognitive, motor and neurological abnormalities (cf. Jamal et al., 1996; Rosenstock et al., 1991). However, it is incomplete as a full account of the symptoms of GWS and supplementary processes are required to explain the diversity of symptoms and their persistence. A recent review of interleukin-1 (IL-1) mediated physiological and behavioural responses by Maier \& Watkins (1998) provides a possible basis for explaining (additional) symptoms not explained by the OPIDP model and it is to this account that we now turn. 


\section{The sickness response}

Maier \& Watkins (1998) propose, and provide ample empirical evidence, that the circuitry of the non-specific immune response can be activated by both stressors and infectious agents, leading to a set of characteristic reactions termed the 'sickness and acute phase response' or 'sickness response'. Non-specific immunity is, after the skin and cilia, the first major line of immunological defence. It responds to general features of identifying 'non-self' and is different from specific immunity which involves the recognition of specific antigens by immunoglobulins and targeting with cytotoxic T-cells and helper T-cells (see Kirkwood \& Lewis, 1989; Weir, 1988). Macrophages are one of the main components of the non-specific immune response, and, in addition to clearing up injured cells, they release pro-inflammatory cytokines. Of these cytokines the most potent is IL-1, to which Maier \& Watkins (1998) attribute the 'sickness response'. They argue that IL-1 operates through bi-directional linkage with the brain to produce an array of cognitive and behavioural changes. They identify the vagus nerve as the means by which IL-1 communicates with the brain. They further show how stressor responses can be attenuated or augmented by the application of IL-1 antagonists or agonists. Based on an accumulation of experimental evidence they state ' ... that many of the sequelae of exposure to stressors are products of these circuits and can better be understood by viewing them as reactions that originally evolved to combat infection and injury' (Maier \& Watkins, 1998, p. 84) and specifically that it is IL-1 that is the important biological mediator.

Table 3 contains the symptoms of GWS and the behavioural and physiological responses seen in the 'sickness response' as defined by Maier \& Watkins (1998). Table 3 shows that almost all of the physiological and behavioural changes attributable to the sickness response are also found in GWS veterans. This remarkable correspondence indicates that the sickness response, which is attributable to IL-1 activity may help to explain the diversity of the symptoms associated with GWS. In short, the mechanisms proposed for the sickness response may also mediate the collection of adverse reactions known as GWS.

If the mechanisms proposed for the sickness response are (at least in part) responsible for the symptoms of GWS, it follows that any treatment for the sickness response should also alleviate aspects of GWS. Pre-clinical testing (on animals) suggests that IL-1 antagonists should be effective against inflammatory diseases like rheumatoid arthritis and stress-related diseases like depression (Dinarello, 1994; Maier \& Watkins, 1998). Whilst evidence from clinical trials on humans is as yet scarce (see Dinarello, 1994; Kent, Bluthe, Kelley \& Dantzer, 1992; Maier \& Watkins, 1998), at least two clinical trials with an IL-1 antagonist (IL-1Ra) have been conducted (see Dinarello, 1994; Granowitz et al., 1992 a b). Thus the clinical evidence to date suggests that IL-1Ra can be used effectively in humans, with no reported adverse effects. Whilst further clinical trials examining dose-response curves, half lives and toxicity are doubtless required, the data to date support the view that treatment with an IL-1 antagonist is a reasonable therapeutic approach to pursue with GWS.

The persistence of these adverse reactions in the home environment could be attributable to the conditioning of this IL-1 mediated sickness response to the smell 
Table 3. Correspondence between GWS symptoms and the sickness response

Sickness and the acute phase response ${ }^{a}$

GWS symptoms/experiences ${ }^{a}$

Physiological adjustments

Fever

Increase in liver metabolism

Alteration in plasma iron, zinc and copper

Leukocytosis

Sleep disturbances

Increased slow wave sleep

Behavioural adjustment

Reduced food and water intake

Reduced activity and exploration

Reduced social behaviour, sexual

behaviour and aggression

Increased pain sensitivity

Depressed mood

Cognitive alteration

Stress response

Increased SNS activity

Increased HPA activity

YES

YES (Milner et al., 1994)

YES

(cf. depression)

Fatigue (47\%)

(cf. depression)

Joint pain $(49 \%)$, muscle pain $(21 \%)$, abdominal pain (17\%), back pain (2\%), headache (39\%)

Depression (23\%)

Memory loss (34\%)

Difficulty concentrating $(27 \%)$

Anxiety states (2.6\%), Syncope

$(2.2 \%)$

a Sickness response (Maier \& Watkins, 1998). GWS symptoms (Comprehensive Clinical Program for Persian Gulf War veterans: Department of Defence report on 18598 participants, 2 April 1996). SNS = increased sympathetic nervous system activity; HPA = increased hypothalmo-pituitaryadrenal activity; $-=$ no data available.

of war and associated experiences. Interest in associative responses has a long history. Pavlov's discovery of classical conditioning was sparked by the observation that, on regular feeding, his dogs came to lick him (as if he were food) at dinner time. Classical conditioning is already known to provide the mechanism for learning based on aversion, e.g. conditioned stimuli for shock (unconditioned stimulus) result in appropriate conditioned responses (CRs) of avoidance and opiate release (Drugan \& Maier, 1983). It has since been discovered that a wide range of our psychological and physiological processes are modulated by this kind of associative process. Indeed, there is already evidence that aspects of the IL-1 circuitry are conditionable (Pezzone, Lee, Hoffman, Pezzone \& Rabin, 1993; Schafe, Seeley \& Bernstein, 1995). Similarly, a variety of immune system reactions can be conditioned to environmental stimuli, including smell (e.g. Ader \& Cohen, 1982), raising the possibility that taste aversion contributes to the strength of conditioned responding (see below). Taste aversion would then be an instance of conditioned sickness. However, this does not exclude the possibility that other physiological, behavioural and psychological aspects of the sickness response could also be conditioned. 


\section{An associative account of GWS}

Gulf war veterans reported that they were exposed to a wide variety of noxious stimuli (see Department of Defence, 1996). These stimuli fall into two broad groupings: (1) fumes, e.g. oil fire smoke (71\%) and (2) psychological stressors, e.g. witnessed casualty $(56 \%)$. Thus a variety of environmental stimuli could have acted as conditioned (CS) and unconditioned stimuli (UCS). UCSs would be attributes of agents causing illness directly (the unconditioned response, UCR) whilst a variety of CSs would be linked with illness through associative learning. Department of Defence data also show that veterans reported that they were subject to a wide variety of direct physiological challenges resulting from exposure to: pyridostigmine bromide $(74 \%)$, pesticides $(66 \%)$, immunization $(49 \%)$, microwaves $(34 \%)$ and depleted uranium $(15 \%)$. The presence of a wide variety of physiological challenges would have cumulated to enhance a non-specific immunological reaction (part of the sickness response) as the initial UCR. Repeated pairings of the available CSs with the UCS and UCR would mean that the sickness response would come to be elicited by the smells, tastes and the sounds of the war. In fact associations based on taste aversion experiments require only a single CS $\rightarrow$ UCS pairing and animals are able to bridge very long delays between CS and UCS presentation. Taste aversion is a special 'biologically prepared' form of learning that extends to other stimuli around when illness is induced (see below).

Most of what we experience as 'taste' (strictly flavour) is actually mediated by odour: foods and other substances reliably taste like they smell. In the case of GWS, we suggest that the smell and taste of war quite literally became associated with the illness and nausea produced by either treatment with immunosuppressive drugs, extreme stress and trauma, or some combination of these factors. For example, a report on one veteran states that '... he now gets a headache any time he smells petroleum. For 13 years before the war he had been a diesel mechanic. Only since the
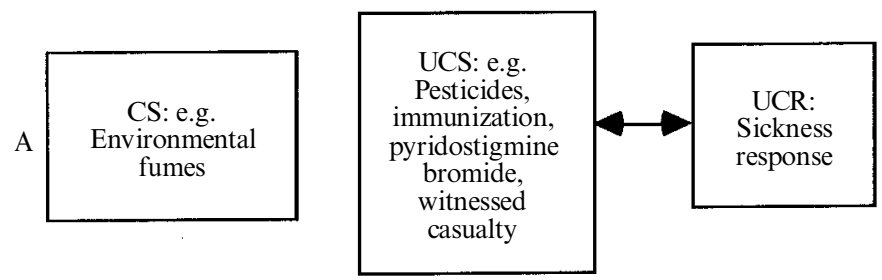

B

CS: e.g. Fumes

CR: Sickness response

Figure 1. An associative mechanism for Gulf War Syndrome. 
war has the smell bothered him. His ears ring and he is constantly tired' (Washington Post, 15 August 1994). This basic model is presented in Fig. 1.

Odour/taste is a particularly potent CS in this context as it has been shown to affect recall of memories (Aggleton \& Waskett, 1999; Parker \& Gellatly, 1997; Schab, 1990) and support conditioned immunological responses (Ader \& Cohen, 1993). Taste aversion has also been demonstrated to occur in response to traumatic treatments likely to result in nausea (see below). Thus psychobiological effects mediated by taste aversion and conditioned illness may be accompanied by memories of the associated experiences. As required by our model, under experimental conditions, cytokines can induce conditioned taste aversion (Goehler et al., 1995). In GWS, re-exposure to the smells of war may trigger: (1) conditioned immune/sickness reactions; and (2) more speculatively, conditioned hyperalgesia (increased sensitivity to pain). Specific aspects of this account (taste aversion, conditioned sickness reactions, conditioned hyperalgesia and a cognitive component) are now explored in more detail.

\section{Taste aversion}

In taste aversion experiments, it has been shown that associating flavour with illness is a very robust form of learning, requiring only a single CS $\rightarrow$ UCS pairing and animals are able to bridge delays between CS and UCS presentation much longer than those needed for other kinds of learning (e.g. Smith \& Roll, 1967). Taste aversion learning seems to be biologically prepared in that flavours are not readily associated with consequences that do not involve illness (e.g. electric shock for rats; Garcia \& Koelling, 1966). However, it has been demonstrated to occur in response to traumatic treatments likely to result in nausea (e.g. centrifugal spin for rats; Hutchison, 1973). Furthermore it is not just flavours that can be associated with illness over long delays : animals will also show aversion to visual features of the food or other stimuli which were around when illness was induced (e.g. a drinking bowl; Revusky \& Parker, 1976). In GWS, conditioned responses could thus include nausea and anxiety, as well as biological changes like reduced immune function. This analysis is consistent with the case described above, in which a range of symptoms were reliably triggered by the smell of petrol. In short, taste and smell stimuli could be both powerful reminders of stress and illness and very effective triggers for conditioned immune and/or sickness responses. We propose that this associative mechanism provides some account of the persistence of GWS.

\section{Conditioned immune/sickness reactions}

While the sickness response is founded on non-specific immunity, research findings from work on acquired (or specific) immunity provides strong evidence for the role of psychological factors in immuno-competence. Acquired immunity has two components: (1) the recognition of foreign material (antigens); and (2) destruction of antigens with antibodies (proteins that recognize antigens and help to kill the invading pathogen). This acquired immunity means that on future exposures, the antigens will be dealt with more rapidly.

We know that the brain influences immunity because stress may (1) increase 
likelihood of infection; (2) aggravate autoimmune diseases; and (3) affect growth of cancers. These effects are likely to be mediated by (1) glucocorticoid secretion (stress hormones are known to suppress the immune system) and (2) via the peripheral nervous system (sympathetic nerve terminals in immune organs like thymus, bone marrow, spleen and lymph nodes). For major reviews on the role of psychological factors on immuno-regulation and disease, reference should be made to the following texts: Andersen, Kiecolt-Glaser \& Glaser, 1994; Cohen \& Williamson, 1991; Maier, Watkins \& Flesher, 1994; Weisse, 1992. Again the contribution of associative processes has been demonstrated both in controlled animal experiments and human clinical studies (see Ader \& Cohen, 1993 for a review). For example, in rats, when a taste (CS) was repeatedly paired with an immuno-suppressive drug (UCS), the taste came to result in a CR like the UCR of immuno-suppression, measured as suppressed antibody response to an antigen (Ader \& Cohen, 1993; Cohen, Moynihan \& Ader, 1994). Similarly, in genetically prone mice, a CS (saccharine flavoured water) was shown to substitute for an immuno-suppressive drug in delaying the onset of an autoimmune disease. This only worked if the saccharine taste had previously been paired with the immuno-suppressive drug to establish it as an effective CS (Ader \& Cohen, 1982). In the clinic, data similarly demonstrate that immune system parameters can be conditioned to the environment in which chemotherapy is administered and immuno-suppression supports conditioned taste aversion (Bovbjerg et al., 1990; Fredrikson et al., 1993).

We do not claim that the symptoms of GWS are equally susceptible to modulation by associative effects, and would not necessarily expect complete overlap between conditioned sickness responses (Maier \& Watkins, 1998) and the symptoms of GWS. Some of the components of the sickness response will be less susceptible to associative effects and will only ever be seen acutely; and some of the additional symptoms of GWS (not seen in the sickness response) may be attributable to additional associative effects such as taste aversion, supported by other sorts of UCS, and other aversive conditioned responses. That said the correspondence between the symptoms of GWS and the sickness response is, in fact, remarkably close (Table 3).

\section{Conditioned byperalgesia}

The joint and muscle pain characteristic of GWS (and the sickness response) might be due to anxiety, whether conditioned or simply persistent in the face of continuing illness. Alternatively, it is conceivable that, instead of the adaptive response of conditioned analgesia to stimuli previously associated with pain, these pains are an opposing counter reaction of hyperalgesia, perhaps following on from the former analgesic CR (Hendrie, 1989). Stress-induced analgesia has been argued to be adaptive in that it allows fight-flight behaviour (Bolles \& Fanselow, 1980). Later hyperalgesia during recovery from injury may be useful to direct attention to the wound site. When such effects become conditioned, we will find that an old war wound hurts most in the presence of associated stimuli. Hyperalgesia is part of the sickness response (Maier, Watkins \& Fleshner, 1994) and pain sensitivity is further increased by peripheral administration of IL-1 (Watkins, Maier \& Goehler, 1995). There is currently no direct evidence to support the speculation that conditioned 
hyperalgesia accounts for any of the symptoms of GWS. But if such a reaction does in fact contribute to the joint and muscle pain characteristic of GWS, it should be blocked by treatment with an IL-1 antagonist. This prediction of our model is clearly testable. The animal work also suggests that conditioned hyperalgesia is naloxonesensitive so (parodoxically) it may also be reduced by treatment with an opiate antagonist (perhaps because it is mediated by an endogenous 'opioid inverse agonist'; Hendrie, 1989).

\section{Cognitive compounding}

Thus we propose that GWS has a psychobiological aspect in that the sickness response can be reinstated in the home environment by stimuli (at least similar to those) that coincided with psychological and physiological stressors in the Gulf. This is not to say that GWS reduces to a 'psychological' learned response. Rather, we suggest that associative triggers modulate both psychological and physiological mechanisms to produce a variety of symptoms, that in the case of GWS, turn out to be components of the sickness response. In addition to their psychobiological effects, these same environmental triggers may also have potent effects on memory because they act as reminders.

Stimuli provided by an environmental context in some way similar to that of the original experience may inevitably precipitate memories. Smell and/or taste stimuli are particularly evocative. For example, in the famous episode of 'la madeleine', Proust, in Remembrance of things past, shows how when memories have faded and their objects have disappeared, the past may often be recalled through smell and taste. Under experimental conditions, this kind of effect is particularly apparent for material or experiences encoded unintentionally into long-term memory in the presence of a predominating odour (see e.g. Aggleton \& Waskett, 1999; Parker \& Gellatly, 1997; Schab, 1990). In the case of GWS, we see memories of the original experience as cognitive responses that may accompany other behavioural and physiological responses.

Thus psychobiological effects mediated by taste aversion, conditioned immune responses and other aspects of the sickness response and/or hyperalgesia may be accompanied by memories of the associated experiences. In a vicious cycle, this would further strengthen the connection between feelings of sickness and thoughts of the Gulf.

\section{Associative mechanisms for other 'syndromes'}

Associative triggers can be extremely powerful and conditioning analyses have had an impact in a number of other clinical disorders: anxiety disorders (following Watson, 1924), drug addiction and epilepsy. Whilst it soon became clear that the objects of fear are no random set (Valentine, 1930), undermining a simple conditioning explanation of their aetiology (see e.g. Gray, 1987, for review), some highly effective behavioural treatments for anxiety disorders show that conditioning principles can nevertheless provide a good account of how fears may be extinguished.

Similarly, there is a substantial literature on the role of associative processes in 
maintaining drug addiction (e.g. O’Brien, Testa, O’Brien, Brady \& Wells, 1977). For example, addicts used to injecting in public lavatories report that lavatory smell elicits craving (Teasdale, 1973); addicts shown a video of heroin preparation and administration report anxiety and craving (Sideroff \& Jarvik, 1980). Conditioned responses can be compensatory to the effects of the drug (antagonistic effects, see Siegel, 1977) or in the same direction (agonistic or incentive effects, see Stewart, de Wit \& Eikelboom, 1984). Whatever the direction of conditioned effects, in extinction procedures, behaviour therapy treatments work by neutralizing the conditioned cues.

Less well known, is that even epilepsy, generally considered a neurological disorder, can be provoked by environmental or psychological triggers and these are very specific and reproducible for the individual concerned (Lishman, 1987). Thus there is already very good evidence that CRs provide some mechanism for a number of clinically significant psychobiological effects and that behavioural treatments provide effective ways to eliminate unwanted reactions. Systematic desensitization using repeated stroboscopic stimulation can even work for photosensitive epilepsy (see Lishman, 1987). We now propose that cognitive-behaviour therapy based on extinction should also be applied to identified precipitants of symptom reporting in GWS.

\section{Conclusions and implications}

We draw the following conclusions from this review. First, on the basis of the existing evidence for the OPIDP theory of GWS, we suggest that this cannot provide a complete account. Secondly, on the issue of single versus multiple syndrome(s), we conclude that the evidence is more consistent with a single syndrome. Thirdly, we have proposed a model of GWS based on the conditionability of the IL-1 system. This paper is intended to prompt further investigation of the existing model(s) of GWS and the development of integrative research programmes towards a complete model of GWS. The associative analysis we present here is complementary to other theories such as that based on OPIDP (an additional rather than an alternative account). For example, OPIDP provides an account of neuropsychological deficits whilst our approach provides some explanation of additional symptomatology that may be seen independently of any neuropsychological deficits. The current model is also testable, with important treatment implications, and has the strength that, unlike other approaches, it can account not only for the diversity of the symptoms of GWS, but also their persistence. Recently, another biological hypothesis of GWS has also focused on cytokines (Rook \& Zumla, 1997). This account does not focus specifically on IL-1 but rather on broader cytokine profiles. Our model also differs in that it emphasizes conditioning processes as a maintenance factor.

In sum, this analysis of recent research has a number of theoretical, research and therapeutic implications. From a theoretical aspect a psycho-neuroendocrinological model, explaining how IL-1 systems that are influenced by associative processes, may be used to understand long-term non-specific symptom reporting in other situations. Thus, whilst we have considered only the contribution of the sickness response to GWS, similar analysis may prove fruitful for a range of non-specific disease responses (cf. Kellner, 1990; Lloyd, 1983). Where associative modulation can be demonstrated 
this opens up the possibility of safe and effective treatment using principles already developed in cognitive behaviour therapy.

From a research perspective the analysis offered in this paper suggests the use of symptomatology/disease diaries to see if Gulf War veterans do indeed experience their symptoms in the presence of specific stimuli. Such methods are common for identifying environmental triggers for other diseases. Further, it suggests that those who report suffering from GWS should have elevated levels of IL-1 in their blood compared to Gulf War veterans who do not report GWS symptoms. There is currently no evidence concerning the pattern of relapse and remission in GWS. Concurrent measurement of physiological changes (e.g. of IL-1 and corticoids) and reports of illness severity will tell us how any episodes of relapse and remission are mediated. We predict, therefore, that levels of IL-1 should vary as a function of the severity of the symptomatology experienced. It should also be increased by further exposure to associated CSs.

Finally, the analysis offered here suggests two therapeutic strategies: (1) drug therapy and (2) cognitive-behavioural therapy. In terms of pharmaceutical interventions it follows that the administration of IL-1 antagonists may be a safe and effective drug therapy. Since an endogenous IL-1 antagonist has been identified (Eisenberg et al., 1990) it should be possible to establish a treatment regime without untoward physiological consequences. Secondly, our analysis suggests that conditioned aspects of GWS can be behaviourally extinguished, perhaps with counter-conditioning to further neutralize the stimuli that otherwise reinstate sickness. For this kind of disorder, the counter-conditioning process could be facilitated pharmacologically (by blocking the sickness response using an IL-1 antagonist). Again these implications are directly testable.

\section{Acknowledgements}

We thank Dr Peter Bibby for a critical reading of the manuscript.

\section{References}

Ader, R. \& Cohen, N. (1982). Behaviorally conditioned immunosuppression and murine systemic lupus erythematosus. Science, 215, 1534-1536.

Ader, R. \& Cohen, N. (1993). Psychoneuroimmunology: conditioning and stress. Annual Review of Psychology, 44, 53-85.

Aggleton, J. \& Waskett, L. (1999). The ability of odours to serve as state-dependent cues for real-world memories: Can Viking smells aid the recall of Viking experiences? British Journal of Psychology, 90, 1-7.

Andersen, B., Kiecolt-Glaser, J. \& Glaser, R. (1994). A biobehavioral model of cancer stress and disease course. American Psychologist, 49, 389-404.

Barnes, J. (1961). Psychiatric sequelae of chronic exposure to organophosphorus insecticides. The Lancet, II, 102-103.

Bolles, R. C. \& Fanselow, M. S. (1980). A perceptual defensive-recuperative model of fear and pain. Behavioral and Brain Sciences, 3, 291-323.

Bovbjerg, D., Redd, W., Maier, L., Holland, J., Lesko, L., Niedzwiecki, D., Rubin, S. \& Hakes, T. 
(1990). Anticipatory immune suppression and nausea in women receiving cyclic chemotherapy for ovarian cancer. Journal of Consulting and Clinical Psychology, 58, 153-157.

Cohen, N., Moynihan, J.A. \& Ader, R. (1994). Pavlovian conditioning of the immune system. International Archives of Allergy and Immunology, 105, 101-106.

Cohen, S. \& Williamson, G. (1991). Stress and infectious disease in humans. Psychological Bulletin, 109, 5-24.

De Bleecker, J., De Reuck, J. \& Willems, J. (1992). Neurological aspects of organophosphate poisoning. Clinical Neurology and Neurosurgery, 94, 93-103.

Department of Defence (1996). Comprehensive clinical evaluation program for Persian Gulf War veterans. UK: Department of Defence.

Dinarello, C. (1994). Interleukin-1. In A. Thomson (Ed.), The cytokine handbook, 2nd ed., pp. 31-55. London: Academic Press.

Drugan, R. C. \& Maier, S. F. (1983). Analgesic and opioid involvement in the shock-elicited activity and escape deficits produced by inescapable shock. Learning and Motivation, 14, 30-47.

Eisenberg, S., Evans, R., Arend, W., Verderber, E., Brewer, M., Hannum, C. \& Thompson, R. (1990). Primary structure and functional expression from complementary DNA of a human interleukin-1 receptor antagonist. Nature, 343, 341-345.

Elderkin-Thompson, V., Silver, R. \& Waitzkin, H. (1998). Narratives of somatizing and nonsomatizing patients in a primary care setting. Journal of Health Psychology, 3, 407-428.

Ferguson, E. (1999). Is there a Gulf War syndrome? The Lancet, 353, 1182.

Ferguson, E. \& Cox, T. (1993). Exploratory factor analysis: A user's guide. International Journal of Selection and Assessment, 1, 84-94.

Fredrikson, M., Hursti, T., Salmi, P., Borjeson, S., Furst, C., Peterson, C. \& Steineck, G. (1993). Conditioned nausea after cancer chemotherapy and autonomic nervous system conditionability. Scandinavian Journal of Psychology, 34, 318-327.

Garcia, J. \& Koelling, R. (1966). Relation of cue to consequence in avoidance learning. Psychonomic Science, 4, 123-124.

Gershon, S. \& Shaw, F. (1961). Psychiatric sequelae of chronic exposure to organophosphorus insecticides. The Lancet, I, 1371-1374.

Goehler, L., Busch, C., Tartaglia, N., Relton, J., Sisj, D., Maier, S. \& Watkins, L. (1995). Blockade of cytokine induced conditioned taste aversion by subdiaphragmatic vagotomy: Further evidence for vagal mediation of immune-brain communication. Neuroscience Letters, 185, 163-166.

Gorsuch, R. (1983). Factor analysis. London: Lawrence Erlbaum.

Granowitz, E., Porat, R., Mier, J. Orencole, S., Callhan, M., Cannon, J., Lynch, E., Ye, K., Poutsiaka, S., Vannier, E., Shapero, L., Pribble, J., Stiles, D., Catalano, M., Wolf, S. \& Dinarello, C. (1992a). Interleukin-1 receptor antagonist blocks the neutrophillia and immunosuppressive effects of experimental endotoxemia. Clinical Research, 40, 651A.

Granowitz, E., Porat, R., Mier, J., Pribble, J., Stiles, D., Bloedow, D., Catalano, M., Wolff, S. \& Dinarello, C. (1992b). Pharmacokinetics, safety and immunomodulatory effects of human recombinant interleukin-1 receptor antagonist in healthy humans. Cytokines, 4, 353-360.

Gray, J. A. (1987). The psychology of fear and stress, 2nd ed., pp. 5-26. Cambridge: Cambridge University Press.

Haley, R., Hom, J., Roland, P., Bryan, W., Van Ness, P., Bonte, F., Devous, M., Mathew, D., Fleckenstein, J., Wians, F., Wolfe, G. \& Kurt, T. (1997a). Evaluation of neurological function in gulf war veterans. Journal of the American Medical Association, 277, 223-230.

Haley, R. \& Kurt, T. (1997). Self-reported exposure to neurotoxic chemical combinations in the gulf war. Journal of the American Medical Association, 277, 2231-2237.

Haley, R., Kurt, T. \& Hom, J. (1997b). Is there a Gulf War Syndrome? Journal of the American Medical Association, 277, 215-222.

Hendrie, C. A. (1989). Naloxone-sensitive hyperalgesia follows analgesia induced by morphine and environmental stimulation. Pharmacology, Biochemistry, and Behaviour, 32, 961-966.

Holden, C. (1997). Evidence for a Gulf War Syndrome? Science, 275, 313.

Hom, J., Haley, R. \& Kurt, T. (1997). Neuropsychological correlates of Gulf War Syndrome. Archives of Clinical Neuropsychology, 12, 531-544. 
Horn, J. (1965). A rationale and test for the number of factors in factor analysis. Psychometricka, 30, 179-185.

Hutchison, S. (1973). Taste aversion in albino rats using centrifugal spin as an unconditioned stimulus. Psychological Reports, 33, 467-470.

Ismail, K., Everitt, B., Blatchley, N., Hull, L., Unwin, C., David, A. \& Wessely, S. (1999). Is there a Gulf War Syndrome? The Lancet 353, 179-182.

Jamal, G. (1995). Long term neurotoxic effects of organophosphate compounds. Adverse Drug Reactions and Toxicological Review, 14, 85-99.

Jamal, G., Hansen, S., Apartopoulos, F. \& Peden, A. (1996). The 'Gulf War syndrome'. Is there evidence of dysfunction in the nervous system? Journal of Neurology, Neurosurgery, and Psychiatry, 60, 449-451.

Kellner, R. (1990). Somatization: Theories and research. Journal of Nervous and Mental Disease, 178, 150-160.

Kendell, R. (1989). Clinical validity. Psychological Medicine, 19, 45-55.

Kent, S., Bluthe, M.-R., Kelley, K. \& Dantzer, R. (1992). Sickness behavior as a new target for drug development. Trends in Pharmaceutical Science, 13, 24-28.

Kimble, R., Matayoshi, A., Vannice, J., Kung, V., Williams, C. \& Pacifici, R. (1995). Simultaneous block of interleukin-1 and tumor necrosis factor is required to completely prevent bone loss in early postovariectomy period. Endocrinology, 136, 3054-3061.

Kirkwood, E. \& Lewis, C. (1989). Understanding medical immunology. Chichester: Wiley.

Lishman, W. A. (1987). Organic psychiatry: The psychological consequences of cerebral disorder, $2 \mathrm{nd}$ ed., pp. 207-276. Oxford: Blackwell Science.

Lloyd, G. (1983). Medicine without signs. British Medical Journal, 287, 539-542.

Lotti, M. (1992). The pathogenesis of organophosphate polyneuropathy. Critical Reviews in Toxicology, $21,465-487$.

Lotti, M., Becker, C. \& Aminoff, M. (1984). Organophosphate polyneuropathy: Pathogenesis and prevention. Neurology, 34, 658-662.

Maier, S. \& Watkins, L. (1998). Cytokines for psychologists: Implications of bidirectional immune-tobrain communication for understanding behavior, mood and cognition. Psychological Bulletin, 105, 83-107.

Maier, S., Watkins, L. \& Flesher, M. (1994). Psychoneuroimmunology: The interface between behavior, brain and immunity. American Psychologist, 49, 1004-1017.

Milner, I., Axelrod, B., Pasquantonio, J. \& Sillanpaa, M. (1994). Is there a gulf war syndrome? Journal of the American Medical Association, 271, 661.

O’Brien, C. P., Testa, T., O’Brien, T. J., Brady, J. P. \& Wells, B. (1977). Conditioned narcotic withdrawal in humans. Science, 195, 1000-1002.

Parker, A. \& Gellatly, A. (1997). Moveable cues: A practical method for reducing context-dependent forgetting. Applied Cognitive Psychology, 11, 163-173.

Petty, C. (1958). Organic phosphate insecticides poisoning. American Journal of Medicine, 43, 467-470.

Pezzone, M., Lee, S., Hoffman, G., Pezzone, K. \& Rabin, B. (1993). Activation of brainstem catecholaminergic neurones by conditioned and unconditioned aversive stimuli as revealed by c-Fos immunoreactivity. Brain Research, 608, 310-318.

Revusky, S. \& Parker, L. (1976). Aversion to unflavored water and cup drinking produced by delayed sickness. Journal of Experimental Psychology (Animal Behavior Processes), 2, 342-353.

Robins, J. \& Kirmayer, L. (1991). Attributions of common somatic symptoms. Psychological Medicine, 21, 1029-1045.

Rook, G. \& Zumla, A. (1997). Gulf war syndrome: is it due to a systemic shift in cytokine balance towards a Th2 profile. Lancet, 349, 1831-1833.

Rosenstock, L., Keifer, M., Daniell, W., McConnell, R. \& Claypoole, K. (1991). Chronic central nervous system effects of acute organophosphate pesticide intoxication. The Lancet, 338, 223-227.

Schab, F. (1990). Odors and the remembrance of things past. Journal of Experimental Psychology: Learning, Memory and Cognition, 16, 648-655.

Schafe, G., Seeley, R. \& Bernstein, L. (1995). Forebrain contribution to the induction of a cellular correlate of conditioned taste aversion in the nucleus of the solitary tract. Journal of Neuroscience, 15 , 6789-6795. 
Sideroff, S. I. \& Jarvik, M. E. (1980). Conditioned responses to a videotape showing heroin-related stimuli. International Journal of the Addictions, 15, 529-536.

Siegel, S. (1977). Morphine tolerance acquistion as an associative process. Journal of Experimental Psychology (Animal Behaviour Processes), 3, 1-13.

Skelton, J. \& Pennebaker, J. (1982). The psychology of physical symptoms and sensations. In G. Sanders \& J. Suls (Eds), Social psychology of health and illness, pp. 99-128. Hillsdale, NJ : Lawrence Erlbaum.

Smith, J. C. \& Roll, D. L. (1967). Trace conditioning with X-rays as an aversive stimulus. Psychonomic Science, 9, 11-12.

Stewart, J., de Wit, H. \& Eikelboom, R. (1984). Role of unconditioned and conditioned drug effects in the self-administration of opiates and stimulants. Psychological Review, 91, 251-268.

Stoller, A., Krupinski, J., Christophers, A. \& Blanks, G. (1965). Organophosphorus insecticides and major mental illness. The Lancet, I, 1387-1388.

Taylor, S. (1983). Adjustment to threatening events: a theory of cognitive adaptation. American Psychologist, 41, 1161-1173.

Teasdale, J. D. (1973). Conditioned abstinence in narcotic addicts. International Journal of the Addictions, 8, 273-292.

Unwin, C., Blatchley, N., Coker, W., Ferry, S., Hotopf, M., Hull, L., Ismail, K., Palmer, I., David, A. \& Wessley, S. (1999). Health of UK servicemen who served in Persian Gulf War. The Lancet, 353, $169-178$.

Valentine, C. W. (1930). The innate bases of fear. Journal of Genetic Psychology, 37, 394-420.

Washington Post (1994). An invisible enemy: The cause of 'Gulf War syndrome' is as elusive as its treatment. 15 August 1994.

Watkins, L., Maier, S. \& Goehler, L. (1995). Immune activation: The role of proinflammatory cytokines in inflammation, illness responses, and pathological pain states. Pain, 63, 289-302.

Watson, J. B. (1924). Behaviorism. New York: Norton.

Weir, D. (1988). Immunology. New York: Churchill Livingstone.

Weisse, C. (1992). Depression and immunocompetence: A review of the literature. Psychological Bulletin, 111, 475-489.

Zwick, W. \& Velicer, W. (1986). Comparison of five rules for determining the number of components to retain. Psychological Bulletin, 99, 432-442. 